Imperial/TP/93-94/51

UCSBTH-94-24

hep-th/9408040

\title{
Extremal black holes as exact string solutions
}

\author{
Gary T. Horowitz* \\ Physics Department \\ University of California, Santa Barbara, CA 93106, USA \\ and \\ A.A. Tseytlin ${ }^{\star \dagger}$ \\ Theoretical Physics Group, Blackett Laboratory \\ Imperial College, London SWr 2BZ, U.K.
}

\begin{abstract}
We show that the leading order solution describing an extremal electrically charged black hole in string theory is, in fact, an exact solution to all orders in $\alpha^{\prime}$ when interpreted in a Kaluza-Klein fashion. This follows from the observation that it can be obtained via dimensional reduction from a five dimensional background which is proved to be an exact string solution.
\end{abstract}

August 1994

\footnotetext{
* e-mail address: gary@cosmic.physics.ucsb.edu

* e-mail address: tseytlin@ic.ac.uk

$\dagger$ On leave from Lebedev Physics Institute, Moscow, Russia.
} 
One of the goals of string theory for many years has been to find exact black hole solutions in four dimensions. The first nontrivial string $\left(\alpha^{\prime}\right)$ corrections to the Schwarzschild solution have been investigated [1], and solutions to the leading order equations describing charged black holes have been found [2,3, 3]. Exact black hole solutions have been constructed in two [5] and three [6] dimensions using (gauged) WZW models based on the group $S L(2, R)$. In addition, using the result that the extremal limit of a black five brane [7] is an exact superstring solution [8], one can (trivially) dimensionally reduce to obtain an exact five dimensional extreme black hole. This black hole has a magnetic type of charge associated with the antisymmetric tensor field. It has recently been shown that one can also dimensionally reduce the exact solution in [8] down to four dimensions [9] to obtain an extreme magnetically charged black hole 10.10

We shall present an exact solution describing an extremal four dimensional electrically charged black hole in (super)string theory. There are several important differences between this and earlier work. First, the electrically charged solutions are qualitatively different from their magnetic analogues. The extremal magnetically charged solutions found in four and five dimensions are products of a timelike line and a euclidean solution. So the lorentzian nature of the solution plays no role. The extremal electrically charged solution, on the other hand, has a nontrivial timelike direction. Second, the previous four and five dimensional extreme black holes were shown to be exact solutions only in the superstring (or heterotic string) theory. Extended supersymmetry (on the world-sheet related to that on space-time) played a key role in the argument [8] that there are no $\alpha^{\prime}$-corrections to the leading-order backgrounds. The solution we will discuss, like the previous two and threedimensional examples, is exact already in the bosonic string theory.

1 These exact five and four dimensional black hole solutions are asymptotically flat. In addition, there are coset CFT or gauged WZW constructions of just the 'throat' regions of some four dimensional extreme magnetically charged dilatonic black holes [11. The fact that the form of the throat solution is unchanged under string corrections was noticed earlier [12]. 
The extremal electric black holes we shall consider are known to be supersymmetric [13] and can be lifted to ten dimensions in such a way that they are dual to special plane fronted waves [14]. However, a priori these properties by themselves are not sufficient to establish that extreme black holes are solutions to all orders in $\alpha^{\prime}$. We will see that another special property of the extremal electric black holes (related to a particular chiral coupling of the string to the background) can be used to show that they are exact solutions.

To satisfy the constraint on the central charge, every solution with an asymptotically flat four dimensional space-time must have a number of internal dimensions. One could assume that the full solution is a simple product of the four dimensional one with a small torus. However, one could equally well assume a tilted product in which off diagonal components of the higher dimensional fields give rise to four dimensional gauge fields. This is the approach we will adopt. We will, in fact, consider mainly the closed bosonic string theory which has no fundamental gauge fields in the higher dimensional space.

It suffices to consider one nontrivial extra dimension (with the remaining ones taken to be a trivial product). We will show that a particular five dimensional bosonic string background is an exact solution, and that its dimensional reduction yields

$$
\begin{gathered}
d s^{2}=-F^{2}(r) d t^{2}+d r^{2}+r^{2} d \Omega, \quad A_{t}=F(r), \\
\phi(r)=\phi_{0}+\frac{1}{2} \ln F(r), \quad F^{-1}(r)=1+\frac{M}{r},
\end{gathered}
$$

where $d \Omega$ denotes the metric of a unit 2-sphere, $A_{\mu}$ is the gauge field potential, $\phi$ is the dilaton and $M$ is twice the ADM mass. This background is precisely the extremal limit of the known leading order solution describing an electric dilaton black hole. In this sense one can say that the leading order extremal black hole solution is exact.

2 Due to the well known ambiguity in the form of the higher order terms in the string equations of motion, the phrase "exact solution" will be understood to mean "exact in a particular renormalization scheme". Different schemes are equivalent in the sense that they are related by local field redefinitions which do not change the physical $S$-matrix. 
Let us first describe a simple application of using dimensional reduction to find exact extremal black holes in string theory. Consider the five dimensional plane fronted wave

$$
d s^{2}=d u d v+K(r) d u^{2}+d r^{2}+r^{2} d \Omega, \quad K(r)=1+\frac{M}{r} .
$$

This background (with constant dilaton and no additional fields) is an exact solution to bosonic string theory [15]. To reduce to four dimensions with $u$ as the internal coordinate, we rewrite (2) in the form

$$
d s^{2}=K\left(d u+\frac{1}{2} K^{-1} d v\right)^{2}-\frac{1}{4} K^{-1} d v^{2}+d r^{2}+r^{2} d \Omega
$$

Letting $t=v / 2$, the four dimensional (string frame) metric, gauge field and scalar $\left(G_{u u}=\right.$ $\left.e^{-2 \sigma}\right)$ are thus

$$
d s^{2}=-K^{-1}(r) d t^{2}+d r^{2}+r^{2} d \Omega, \quad A_{t}=K^{-1}(r), \quad \sigma=-\frac{1}{2} \ln K(r)
$$

This is just the extremal electrically charged Kaluza-Klein black hole [2,10]. So the extreme Kaluza-Klein black hole can be viewed as an exact string solution.

We have recently shown [16] that a class of solutions to the leading order bosonic string equations are, in fact, exact. Included in this class was the five dimensional fundamental string solution [17]

$$
\begin{aligned}
d s^{2} & =F(r) d u d v+d r^{2}+r^{2} d \Omega \\
B_{u v}=\frac{1}{2} F(r), \quad \phi & =\phi_{0}+\frac{1}{2} \ln F(r), \quad F(r)=\left(1+\frac{M}{r}\right)^{-1},
\end{aligned}
$$

where $B_{\mu \nu}$ is the antisymmetric tensor. If one writes $v=y+t, u=y-t$ and reduces this solution to four dimensions by assuming $y$ to be the internal coordinate, one again obtains the extreme Kaluza-Klein black hole (4) [18]. This is not surprising since the fundamental string solution (5) is related to the plane fronted wave (2) by a spacetime duality transformation [19, and it turns out that the Kaluza-Klein metric is invariant under this duality. The main effect of the duality transformation is that the gauge field 
in four dimensions now comes from the antisymmetric tensor $B_{\mu \nu}$ and not from the offdiagonal components of the metric.

To obtain the extreme black hole (1) in four dimensions, we must start with the following generalization of the fundamental string solution

$$
d s^{2}=F(r) d u d v+d u^{2}+d r^{2}+r^{2} d \Omega
$$

with $B_{u v}, \phi$ and $F$ again given by (6). This background was found to be a solution of the leading order string equations in [20] and was further discussed in [21]. We will show that the arguments in [16] can in fact be extended to establish that (7) is also an exact solution. But first, we describe some properties of this solution and show that its dimensional reduction yields the extreme charged black hole.

Unlike the fundamental string (5), the metric (7) has a regular ergosphere. This can be seen by introducing new coordinates $u=y-t, v=2 t$ so that the metric becomes

$$
d s^{2}=-\left(\frac{r-M}{r+M}\right) d t^{2}-\frac{2 M}{r+M} d t d y+d y^{2}+d r^{2}+r^{2} d \Omega .
$$

It is now clear that the Killing vector $\partial / \partial t$, which is a unit time translation at infinity, becomes null at $r=M$. This surface is an ergosphere and not an event horizon since it is timelike. One can still travel from $r<M$ to $r>M$ provided one moves in the $y$ direction. Although the metric components remain finite at $r=0$, the metric becomes degenerate there and the curvature diverges.

One can understand the fact that (8) has an ergosphere while (5) does not as follows. It is known that the fundamental string is the extremal limit of a two parameter family of charged black string solutions which can be obtained by boosting the direct product of the Schwarzschild background with a line, and applying a duality transformation [7, 19]. The result is $(S \equiv \sinh \alpha, C \equiv \cosh \alpha, \alpha$ is the original boost parameter)

$$
d s^{2}=\left(1+\frac{2 m S^{2}}{r}\right)^{-1}\left[-\left(1-\frac{2 m}{r}\right) d t^{2}+d y^{2}\right]+\left(1-\frac{2 m}{r}\right)^{-1} d r^{2}+r^{2} d \Omega
$$




$$
B_{y t}=\frac{C}{S}\left(1+\frac{2 m S^{2}}{r}\right)^{-1}, \quad e^{-2 \phi}=1+\frac{2 m S^{2}}{r} .
$$

The extremal limit corresponds to sending $m \rightarrow 0, \alpha \rightarrow \infty$ in such a way that $M \equiv 2 m e^{2 \alpha}$ is held fixed. In this limit the horizon at $r=2 m$ shrinks down to zero size and becomes singular. The charged black string solution (9) approaches the fundamental string (5). If we add linear momentum to (9) by applying a boost $t=\hat{t} \cosh \beta+\hat{y} \sinh \beta, \quad y=\hat{t} \sinh \beta+$ $\hat{y} \cosh \beta$, then we create an ergosphere outside the horizon. The extremal limit $m \rightarrow$ $0, \alpha, \beta \rightarrow \infty$ with $M \equiv 2 m e^{2 \alpha}=2 m e^{2 \beta}$ fixed, now yields the generalized fundamental string (8). So this solution can also be viewed as the extremal limit of a charged black string, but now with non-zero linear momentum. The horizon still shrinks to zero size, but the ergosphere remains.

If $y$ is periodically identified with a small period, the generalized fundamental string (8) will appear as four dimensional. We can obtain the effective four dimensional geometry by rewriting (8) in the form

$$
d s^{2}=\left(d y-\frac{M}{r+M} d t\right)^{2}-\frac{r^{2}}{(r+M)^{2}} d t^{2}+d r^{2}+r^{2} d \Omega
$$

Thus the four dimensional metric is

$$
d s^{2}=-\frac{r^{2}}{(r+M)^{2}} d t^{2}+d r^{2}+r^{2} d \Omega
$$

i.e. is precisely that of the extreme electrically charged black hole (11). Note that the dilaton is the same as in five dimensions since the "modulus" field from the dimensional reduction is constant. The gauge field now comes from both the off-diagonal components of the metric and the antisymmetric tensor which are equal (up to a gauge transformation).

We now turn to the demonstration that the background (6),(77) (or (8)) is an exact string solution. The bosonic string in a 'massless' background is described (in the conformal gauge) by the $\sigma$-model

$$
I=\frac{1}{\pi \alpha^{\prime}} \int d^{2} z L, \quad L=\left(G_{\mu \nu}+B_{\mu \nu}\right)(X) \partial X^{\mu} \bar{\partial} X^{\nu}+\alpha^{\prime} \mathcal{R} \phi(X)
$$


where $G_{\mu \nu}$ is the spacetime metric, and $\mathcal{R}$ is related to the world-sheet metric $\gamma$ and its scalar curvature by $\mathcal{R} \equiv \frac{1}{4} \sqrt{\gamma} R^{(2)}$. Consider a $\sigma$-model of the form

$$
L_{F}=F(x) \partial u \bar{\partial} v+\partial x^{i} \bar{\partial} x^{i}+\alpha^{\prime} \mathcal{R} \phi(x) .
$$

It was shown in [16] that this model is conformally invariant to all orders (in a particular scheme) if

$$
\partial^{2} F^{-1}=0, \quad \phi=\phi_{0}+\frac{1}{2} \ln F(x) .
$$

The fundamental string solution corresponds to the case of $F^{-1}=1+M / r$. The key feature which enables one to establish the conformal invariance to all orders is the chiral coupling of the $u$ and $v$ fields to the background, which is obtained by setting $G_{u v}=B_{u v}$. This results in the two chiral currents associated with the null translational symmetries. It turns out that a single chiral current associated with a null symmetry is sufficient to establish the conformal invariance of similar backgrounds to all orders provided the oneloop conditions are satisfied. This means that a much larger class of leading-order solutions can be shown to be exact. The most general situation will be discussed elsewhere [22]. Here we consider the following class of $\sigma$-models which includes (6),(7) as a special case

$$
L_{F K}=F(x) \partial u \bar{\partial} v+K(x) \partial u \bar{\partial} u+\partial x^{i} \bar{\partial} x^{i}+\alpha^{\prime} \mathcal{R} \phi(x) .
$$

To find the exact conditions of conformal invariance we follow [16] by introducing the source terms ( $z$ denotes the two world-sheet coordinates)

$$
L_{\text {source }}=V(z) \partial \bar{\partial} u+U(z) \partial \bar{\partial} v+X(z) \partial \bar{\partial} x,
$$

and performing the path integral over $v$. The resulting $\delta$-function sets $\partial u=F^{-1} \partial U$ (up to a zero mode which we absorb in $U$ ). We arrive at the following effective $x$-theory

$$
\begin{gathered}
L_{F K}^{\prime}=\partial x^{i} \bar{\partial} x^{i}-F^{-1}(x) \partial U \bar{\partial} V \\
+K(x) F^{-1}(x) \partial U \bar{\partial} \partial^{-1}\left[F^{-1}(x) \partial U\right]+\alpha^{\prime} \mathcal{R}\left(\phi-\frac{1}{2} \ln F\right)+X \partial \bar{\partial} x
\end{gathered}
$$


where the shift in $\phi$ comes from the determinant and we make use of a special scheme to keep the free kinetic term of $x^{i}$ unchanged (see [16]). The conditions of conformal invariance of the $O(\partial U \bar{\partial} V)$ term are the same as for the model with $K=0$ (14). The conformal anomaly must be local, so that only the local part of the non-local $O(\partial U \partial U)$ term may contribute to it. Since this non-local term already contains two factors of $\partial U$ it cannot produce $\partial X$-dependent counterterms. That means we may expand the functions $K F^{-1} \equiv \tilde{K}$ and $F^{-1}$ in it near a constant, $x^{i}(z)=x_{0}^{i}+\eta^{i}(z)$

$$
\begin{gathered}
\int d^{2} z d^{2} z^{\prime}[\tilde{K}(x) \partial U](z) \bar{\partial}^{2} \Delta^{-1}\left(z, z^{\prime}\right)\left[F^{-1}(x) \partial U\right]\left(z^{\prime}\right) \\
=\sum_{n, m=0}^{\infty} \frac{1}{n ! m !} \partial_{i_{1}} \ldots \partial_{i_{m}} \tilde{K}\left(x_{0}\right) \partial_{j_{1}} \ldots \partial_{j_{n}} F^{-1}\left(x_{0}\right) \\
\int d^{2} z d^{2} z^{\prime}\left(\eta^{i_{1}} \ldots \eta^{i_{m}}\right)(z) \partial U(z) \bar{\partial}^{2} \Delta^{-1}\left(z, z^{\prime}\right)\left(\eta^{j_{1}} \ldots \eta^{j_{n}}\right)\left(z^{\prime}\right) \partial U\left(z^{\prime}\right)
\end{gathered}
$$

where we defined $\Delta^{-1}$ by $\partial \bar{\partial} \Delta^{-1}=\delta^{(2)}\left(z, z^{\prime}\right)$. Then the only contractions of the quantum fields $\eta^{i}$ that can produce local $O(\partial U \bar{\partial} U)$ divergences are the one-loop tadpoles on the left and right side of the non-local propagator $\Delta^{-1}\left(z, z^{\prime}\right)$. Any contraction between $\eta^{n}(z)$ and $\eta^{m}\left(z^{\prime}\right)$ gives additional $\Delta^{-1}\left(z, z^{\prime}\right)$-factors and thus contributes only to the non-local part of the corresponding $2 d$ effective action.

As a result, we find the following conformal invariance condition

$$
F^{-1} \partial^{2} \tilde{K}+\tilde{K} \partial^{2} F^{-1}=0
$$

or, combined with (14),

$$
\partial^{2} F^{-1}=0, \quad \partial^{2}\left(K F^{-1}\right)=0, \quad \phi=\phi_{0}+\frac{1}{2} \ln F(x) .
$$

When $K=0$ these conditions obviously reduce to (14). When $F=1$ the $\sigma$-model (15) describes the standard plane fronted wave, and (20) gives the usual $\partial^{2} K=0$ condition for this background. The five dimensional solution (7) which yields the charged black hole 
corresponds to the simplest nontrivial generalization (15) of the fundamental string (13) with $K=1$.

This class of solutions is mapped into itself under the duality transformation with respect to a general spacelike translation in the $(u, v)$ plane. Setting $v=\hat{v}+q u(q=$ const) in (15) and dualizing with respect to $u$ yields a $\sigma$-model of exactly the same form with $F, K$, and $\phi$ replaced by

$$
\tilde{F}=\frac{F}{K+q F}, \quad \tilde{K}=\frac{1}{K+q F}, \quad \tilde{\phi}=\phi-\frac{1}{2} \ln (K+q F) .
$$

In other words, the null translational symmetry and chiral coupling are preserved under the duality. The conformal invariance conditions (20) are of course invariant under this transformation.

The above discussion was in the context of the bosonic string theory. A generalization to the case of the closed superstring theory is straightforward. One only has to repeat our arguments starting with the $(1,1)$ supersymmetric extension of the bosonic $\sigma$-model (15) (and to note that the one-loop conformal invariance conditions are the same as in the non-supersymmetric case) with the conclusion that the above bosonic backgrounds represent also the superstring solutions. Moreover, any closed superstring solution can be embedded into the heterotic string theory by identifying the generalized spin connection with a heterotic gauge field background (i.e. by rewriting the $(1,1)$ supersymmetric $\sigma$ model in the $(1,0)$-supersymmetric heterotic form [23]).

Therefore, the exact bosonic solution (6),(17) is also an exact $D=5$ solution of the heterotic string theory. Like the bosonic one, it can also be given a Kaluza-Klein interpretation as a four dimensional extremal electric black hole background. In this case the four dimensional abelian gauge field of the electric dilatonic black hole has a Kaluza-Klein origin, while the role of the heterotic gauge field background is to combine with the spin connection contributions to make the model $(1,1)$ supersymmetric. 3

3 Note that the Yang-Mills $\operatorname{Tr} F^{2}$-term in the four dimensional heterotic string effective action is then treated as an $\alpha^{\prime}$-correction (i.e. on an equal footing with the $R^{2}$-term) while the abelian $F^{2}$-term originating from the $D=5$ scalar curvature term has the same order as the $D=4$ Einstein $R$-term. 
Given a $D=4$ leading-order bosonic background, its embedding into the heterotic string theory is not unique. The embeddings of extremal $D=4$ dilatonic black holes in which the $U(1)$ gauge field has Kaluza-Klein and not heterotic Yang-Mills origin have extended $(N=2, D=4)$ space-time supersymmetry [13]. This suggests that the corresponding world-sheet theory should presumably have $(4,4)$ supersymmetry [24] and thus, as in the case considered in [8], the leading-order higher-dimensional superstring or heterotic string solutions should remain exact (both in the magnetic and electric cases). 1

At the same time, there should also exist a related solution of the heterotic string theory formulated directly in $D=4$. In fact, the charged dilatonic black hole may be considered as a non-supersymmetric leading-order solution of the $D=4$ heterotic string theory with the charge being that of the $U(1)$ subgroup of the Yang-Mills gauge group. This solution must have an extension to higher orders in $\alpha^{\prime}$ which, in general, may not be the same as the above 'Kaluza-Klein' solution. Even though the leading-order terms in the compactified (from $D=5$ to $D=4$ ) bosonic string theory and $D=4$ heterotic string theory with a $U(1)$ gauge field background look the same, the $\alpha^{\prime}$-corrections are different, so that our bosonic result does not automatically imply that the extremal electric black hole considered as a $D=4$ heterotic string solution is also exact. However, given that the bosonic string solution is just the leading-order one when considered in a special scheme, it is an interesting possibility that a similar special scheme exists also in the heterotic case. If true, that would imply that extremal electrically charged black holes remain exact heterotic string solutions even in the case of the non-supersymmetric embedding, 鴫

To summarize, we have found an exact five dimensional string solution (60),(7) which reduces to the extremal electrically charged black hole (11) in four dimensions. Our results further support the special nature of extremal black holes. In particular, the exactness of the leading order extremal electric black hole implies that the extreme charge to mass ratio

\footnotetext{
4 This possibility was advocated to us by A. Strominger.

5 This does not seem to be true for the magnetically charged black hole, which has non-trivial $\alpha^{\prime}$ corrections in the non-supersymmetric case 25].
} 
should not be renormalised by $\alpha^{\prime}$ corrections. Our method does not apply to non-extremal black holes which are likely to have $\alpha^{\prime}$ corrections in all renormalization schemes. (It is easy to show that this is the case for the Schwarzschild solution.)

One of the motivations for having exact black hole solutions is to better understand the behavior of strong gravitational fields and the possible existence of singularities in string theory. Some preliminary observations are the following. In the bosonic string theory the solution appears as four dimensional with a null singularity at low energy. However, as one approaches the singularity, one discovers that the solution is fundamentally the product of a five dimensional solution and a torus. The five dimensional solution has an ergosphere with a curvature singularity inside. Whether this singularity adversely affects string propagation remains to be seen. One should note that the string coupling $e^{\phi}$ goes to zero near the singularity suggesting that quantum corrections will be suppressed.6

As we noted earlier, the methods used here can be applied to a larger class of backgrounds to show that many low energy solutions are, in fact, exact. These include the recently discovered dilatonic IWP metrics [26,27]. The details will be given elsewhere [22].

We wish to thank G. Gibbons, R. Kallosh and A. Strominger for useful and stimulating discussions. Part of this work was done during the LMS Durham Symposium "Quantum concepts in time and space" and we would like to express our gratitude to the organizers of this excellent meeting. G.H. was supported in part by NSF Grant PHY-9008502 and by EPSRC grant GR/J82041. A.A.T. acknowledges the support of PPARC.

6 This can be compared with the extreme Kaluza-Klein black hole (雨) which, as we have seen, is also an exact solution. This solution appears as four dimensional with a timelike (naked) singularity at low energy. But near the singularity one again finds that the solution is actually a product of a torus with a five dimensional solution which has an ergosphere and a singularity inside. However, in this case the string coupling remains constant. 


\section{References}

[1] C. Callan, R. Myers, and M. Perry, Nucl. Phys. B311 (1988) 673.

[2] G. Gibbons, Nucl. Phys. B207 (1982) 337.

[3] G. Gibbons and K. Maeda, Nucl. Phys. B298 (1988) 741.

[4] D. Garfinkle, G. Horowitz and A. Strominger, Phys. Rev. D43 (1991) 3140; D45 (1992) 3888(E).

[5] E. Witten, Phys. Rev. D44 (1991) 314.

[6] G. Horowitz and D. Welch, Phys. Rev. Lett. 71 (1993) 328; N. Kaloper, Phys. Rev. D48 (1993) 2598.

[7] G. Horowitz and A. Strominger, Nucl. Phys. B360 (1991) 197.

[8] C. Callan, J. Harvey and A. Strominger, Nucl. Phys. B359 (1991) 611.

[9] R. Khuri, Nucl. Phys. B387 (1992) 315; Phys. Lett. B294 (1992) 325; J. Gauntlett, J. Harvey and J. Liu, Nucl. Phys. B409 (1993) 363.

[10] M. Duff, R. Khuri, R. Minasyan and J. Rahmfeld, Nucl. Phys. B418 (1994) 195.

[11] S. Giddings, J. Polchinski and A. Strominger, Phys. Rev. D48 (1993) 5784; W. Nelson, Phys. Rev. D49 (1994) 5302; D. Lowe and A. Strominger, "Exact FourDimensional Dyonic Black Holes and Bertotti-Robinson Spacetimes in String Theory", UCSBTH-94-14, hep-th/9403186; C. Johnson, "Exact models of extremal dyonic 4D black hole solutions of heterotic string theory", IASSNS-HEP-94/20, hep-th/9403192.

[12] M. Gürses, Phys. Rev. D46 (1992) 2522.

[13] R. Kallosh, A. Linde, T. Ortín, A. Peet and A. Van Proeyen, Phys. Rev. D46 (1992) 5278.

[14] E. Bergshoeff, R. Kallosh and T. Ortín, "Black-hole-wave duality in string theory", SU-ITP-94-11, hep-th/9406009.

[15] R. Güven, Phys. Lett. B191 (1987) 275; D. Amati and C. Klimčík, Phys. Lett. B219 (1989) 443; G. Horowitz and A. Steif, Phys. Rev. Lett. 64 (1990) 260.

[16] G. Horowitz and A. Tseytlin, "On exact solutions and singularities in string theory", preprint Imperial/TP/93-94/38; hep-th/9406067.

[17] A. Dabholkar, G. Gibbons, J. Harvey and F. Ruiz, Nucl. Phys. B340 (1990) 33.

[18] J. Gauntlett, talk presented at the conference "Quantum Aspects of Black Holes", Santa Barbara, June 1993.

[19] J. Horne, G. Horowitz and A. Steif, Phys. Rev. Lett. 68 (1992) 568.

[20] D. Waldram, Phys. Rev. D47 (1993) 2528.

[21] J. Gauntlett, J. Harvey, M. Robinson and D. Waldram, Nucl. Phys. B411 (1994) 461.

[22] G. Horowitz and A. Tseytlin, "New exact solutions in string theory", in preparation.

[23] A. Sen, Phys. Rev. D32 (1985) 2102; C. Hull and E. Witten, Phys. Lett. B160 (1985) 398. 
[24] T. Banks and L. Dixon, Nucl. Phys. B307 (1988) 93.

[25] M. Natsuume, "Higher order corrections to the GHS string black hole", NSF-ITP-9466, hep-th/9406079.

[26] R. Kallosh, D. Kastor, T. Ortín and T. Torma, "Supersymmetry and stationary solutions in dilaton-axion gravity", SU-ITP-94-12, hep-th/9406059.

[27] C. Johnson and R. Myers, "Taub-NUT Dyons in Heterotic String Theory", IASSNSHEP-94/50, McGill/94-28, hep-th/9406069. 